\title{
Resonant spin orientation at the exciton level anticrossing in InP quantum dots
}

\author{
Yasuaki Masumoto, ${ }^{*}$ Ken Toshiyuki, Tsukasa Suzuki, and Michio Ikezawa \\ Institute of Physics and Center for Tsukuba Advanced Research Alliance (TARA), University of Tsukuba, Tsukuba 305-8571, Japan
}

(Received 28 August 2007; revised manuscript received 22 December 2007; published 18 March 2008)

\begin{abstract}
Resonant spin orientation of excitons was observed under linearly polarized quasiresonant excitation around two anticrossing points of excitons in InP quantum dots (QDs). Under the longitudinal and tilted magnetic fields of 1.5 and $2.5 \mathrm{~T}$, two anticrossings of bright and dark excitons take place. At the anticrossing points, bright and dark excitons mix with each other and the wave function mixing induces the resonant spin orientation. Time-resolved circular polarization clearly showed the resonant spin orientation due to mixing of bright and dark excitons at the anticrossing points. Dark excitons can work as the carrier and spin reservoir because of their long lifetime. We demonstrated that magnetic field can control the spin entanglement and the resonant spin orientation of QDs.
\end{abstract}

DOI: 10.1103/PhysRevB.77.115331

PACS number(s): 78.67.Hc, 71.35.Ji, 72.25.Rb

Spin polarization in semiconductors has attracted considerable attention of researchers in recent years since it is expected to be used in quantum information processing. ${ }^{1}$ Especially, quantum dots (QDs) are promising candidates for quantum bit and quantum spin memory ${ }^{2}$ because spin relaxation is greatly suppressed by three-dimensional quantum confinement of carriers in QDs. ${ }^{3}$ Spin polarization of excitons can be produced either by the spin relaxation in the magnetic field or by optical orientation. In the magnetic field, not only thermal spin orientation among Zeeman sublevels but also the resonant spin orientation due to mixing of Zeeman sublevels can work. The resonant spin orientation due to mixing of exciton sublevels was observed experimentally in type I and II quantum wells ${ }^{4,5}$ but not in QDs.

The lowest-energy exciton in III-V QDs is composed of an electron with the spin angular momentum projection $s_{e z}$ $= \pm \frac{1}{2}$ and a heavy hole with the total angular momentum projection $J_{h z}= \pm \frac{3}{2}$. Combination of $2 \times 2$ spin states gives quartet excitons, $\left|-\frac{1}{2},+\frac{3}{2}\right\rangle,\left|+\frac{1}{2},-\frac{3}{2}\right\rangle,\left|+\frac{1}{2},+\frac{3}{2}\right\rangle,\left|-\frac{1}{2},-\frac{3}{2}\right\rangle$, simply denoted by projection of the total angular momentum of the exciton, $J=s_{e}+J_{h}$, upon the quantization axis, $|m\rangle_{0},(m$ $=+1,-1,+2,-2)$, respectively. Quartet excitons are also denoted by spin projection, $|\downarrow\rangle|\uparrow\rangle,|\uparrow\rangle|\downarrow\rangle,|\uparrow\rangle|\uparrow\rangle,|\downarrow\rangle|\downarrow\rangle$, where the first ket vector represents the electron spin and the second ket vector the hole spin. Bright excitons, $|-1\rangle_{0},|+1\rangle_{0}$, can be directly excited by light and can luminesce. They decay at the radiative decay rate plus the nonradiative decay rate.
Dark excitons, $|+2\rangle_{0},|-2\rangle_{0}$, cannot be directly excited by light nor can luminesce. Dark excitons decay at the nonradiative decay rate and, therefore, usually have a long lifetime.

Spin flip of either an electron or a hole is needed for the conversion between bright excitons and dark excitons. If bright and dark excitons are equally formed and if the spin flip rate is not much faster than the lifetime of the exciton, dark excitons are superior in existing probability to bright excitons. Then, dark excitons can work as the carrier and spin reservoir because of their long lifetime. In fact, four eigenvectors in the tilted magnetic field are represented by the entangled linear combination of $|\uparrow\rangle|\downarrow\rangle,|\downarrow\rangle|\uparrow\rangle,|\uparrow\rangle|\uparrow\rangle$, and $|\downarrow\rangle|\downarrow\rangle$. Magnetic field can mix bright excitons with dark excitons. It can mix resonantly $|\downarrow\rangle|\uparrow\rangle$ with $|\uparrow\rangle|\uparrow\rangle$ or $|\uparrow\rangle|\downarrow\rangle$ with $|\uparrow\rangle|\uparrow\rangle$. Then, dark excitons work as the spin reservoir prominently. In this study, we report the observation of magneticfield-induced anticrossing and state mixing of bright and dark excitons and resonant spin orientation at the anticrossing points under linearly polarized excitation in InP QDs. We demonstrated that magnetic field can control the spin entanglement and the resonant spin orientation of QDs.

Bright and dark exciton states in a magnetic field are calculated in terms of a spin Hamiltonian. ${ }^{6,7}$ A set of four states, $|+1\rangle_{0},|-1\rangle_{0},|+2\rangle_{0}$, and $|-2\rangle_{0}$, specifies bases of matrix representation of the heavy-hole-exciton Hamiltonian consisting of the first exchange interaction term and the second Zeeman term given by

$$
\frac{1}{2}\left(\begin{array}{cccc}
\delta_{0} & \delta_{1} & 0 & 0 \\
\delta_{1} & \delta_{0} & 0 & 0 \\
0 & 0 & -\delta_{0} & \delta_{2} \\
0 & 0 & \delta_{2} & -\delta_{0}
\end{array}\right)+\frac{\mu_{B} B}{2}\left(\begin{array}{c}
\left(-g_{e, z}+g_{h, z}\right) \cos \theta \\
0 \\
g_{e, x} \sin \theta \\
g_{h, x} \sin \theta
\end{array}\right.
$$

if the $g$ factor of electrons and holes in the QDs is axially symmetric. Here, $\theta$ is the angle between the crystal growth axis denoted by the $z$ axis and direction of the magnetic field $B$ lying in the $x-z$ plane. For InP QDs in the present sample,

$$
\left.\begin{array}{ccc}
0 & g_{e, x} \sin \theta & g_{h, x} \sin \theta \\
\left(g_{e, z}-g_{h, z}\right) \cos \theta & g_{h, x} \sin \theta & g_{e, x} \sin \theta \\
g_{h, x} \sin \theta & \left(g_{e, z}+g_{h, z}\right) \cos \theta & 0 \\
g_{e, x} \sin \theta & 0 & -\left(g_{e, z}+g_{h, z}\right) \cos \theta
\end{array}\right)
$$

electron-hole exchange interactions $\left(\delta_{0}=0.141 \mathrm{meV}\right.$, $\delta_{1}=0.038 \mathrm{meV}$, and $\left.\delta_{2}=0\right)$, the electron $g$ factor $\left(g_{e, z}=1.5\right.$ and $\left.g_{e, x}=1.5\right)$, and the hole $g$ factor $\left(g_{h, x}=0.25\right)$ are known from our preceding studies and $g_{h, z}$ is unknown. ${ }^{7-9}$ Exchange 


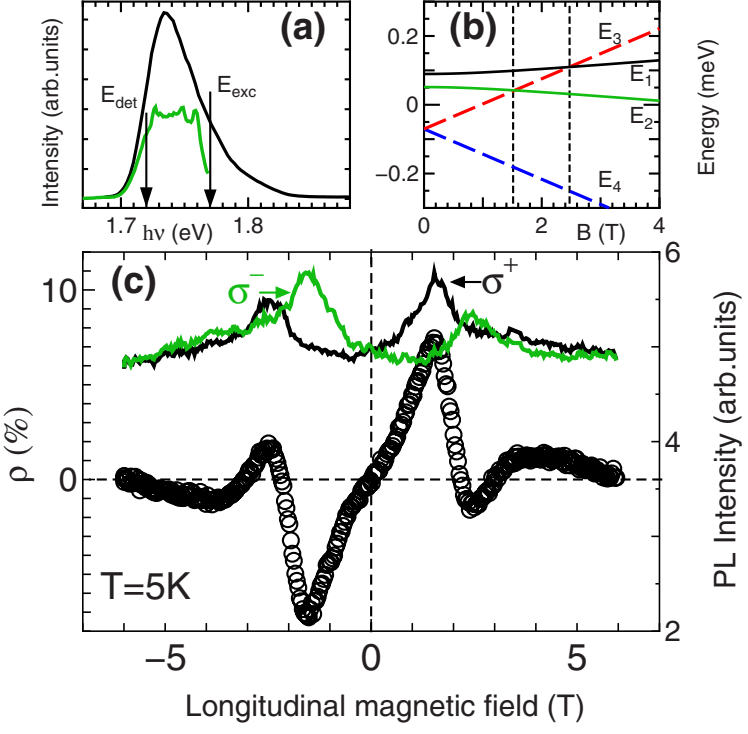

FIG. 1. (Color online) (a) PL spectrum of the sample under $\mathrm{HeNe}$ laser excitation at $1.959 \mathrm{eV}$ and under quasiresonant excitation of $1.771 \mathrm{eV}$. The electric bias of $U=-0.8 \mathrm{~V}$ was applied. (b) Calculated eigenenergies $E_{j}$ for InP QDs studied at $\theta=0^{\circ}$. Here, $g_{h, z}=1.02$ is assumed. (c) Circular polarization $(\bigcirc)$ of PL of neutral InP QDs detected at $E_{\text {det }}=1.722 \mathrm{eV}$ under the longitudinal magnetic field $\left(\theta=0^{\circ}\right)$ at $5 \mathrm{~K}$. Linearly polarized laser light excited InP QDs at $E_{\text {exc }}=1.771 \mathrm{eV}$ quasiresonantly below the $\operatorname{In}_{0.5} \mathrm{Ga}_{0.5} \mathrm{P}$ barrier band gap. The $\sigma^{+}$and $\sigma^{-}$polarized PL intensities are shown as a function of the longitudinal magnetic field.

interaction $\delta_{0}$ splits the bright and dark excitons and $\delta_{1}$ the bright excitons at $B=0 \mathrm{~T}$.

The eigenenergies and eigenvectors are derived by the diagonalization of the real symmetric Hamiltonian matrix. Eigenenergies and eigenvectors are given by $E_{j}$ and $|j\rangle=\Sigma_{m} C_{j m}|m\rangle_{0}(j=1-4)$, respectively, where $C_{j m}$ is real. $|1\rangle,|2\rangle,|3\rangle$, and $|4\rangle$ are named in energy order from top to bottom at $B=0 \mathrm{~T}$. Tilted magnetic field can mix the bright and dark exciton states and cause their anticrossing. In the Faraday configuration $\left(\theta=0^{\circ}\right)$, bright excitons and dark excitons do not mix with each other, and $E_{1,2}=(1 / 2)\left[\delta_{0} \pm \sqrt{\delta_{1}^{2}+\left(g_{h, z}-g_{e, z}\right)^{2} \mu_{B}^{2} B^{2}}\right]$ and $E_{3,4}$ $=(1 / 2)\left[-\delta_{0} \pm\left(g_{h, z}+g_{e, z}\right) \mu_{B} B\right]$ are obtained by the matrix diagonalization. Zeeman energy of the dark exciton is larger than that of bright excitons and hence causes energy crossing. Calculated $E_{1}, E_{2}, E_{3}$, and $E_{4}$ at $\theta=0^{\circ}$ are plotted in Fig. 1(b), where $g_{h, z}=1.02$ is assumed. A dark exciton $|+2\rangle_{0}$ crosses with bright excitons $|-1\rangle_{0}$ and $|+1\rangle_{0}$ at 1.5 and $2.5 \mathrm{~T}$, respectively.

The sample studied was charge-tunable InP selfassembled QDs (areal density $\sim 10^{10} \mathrm{~cm}^{-2}$ ) grown on an $n^{+}$-GaAs substrate. A single layer of InP QDs $40 \mathrm{~nm}$ in average diameter and $5 \mathrm{~nm}$ in height was grown between the $100 \mathrm{~nm} \mathrm{In}_{0.5} \mathrm{Ga}_{0.5} \mathrm{P}$ barrier layers. Under the above-barrier excitation, the sample shows the photoluminescence (PL) band peaked at $1.73 \mathrm{eV}$, as shown in Fig. 1(a). External electric bias applied to the sample controlled the Fermi level of the electron doped substrate and the charged state of the QDs. ${ }^{7,10}$ Trionic quantum beat showed that there is, on an average, one doped electron per dot under electric bias of $U=-0.1 \mathrm{~V} .{ }^{10}$ Under the positive bias, QDs are doped by more than two electrons, while QDs become neutral below $U=-0.4 \mathrm{~V}$, where excitonic quantum beat was clearly observed. $^{7}$

A continious-wave or picosecond Ti:sapphire laser was used for the linearly polarized quasiresonant excitation of InP QDs at $1.771 \mathrm{eV}$. The sample was set in a superconducting magneto-optic cryostat $(|B| \leqslant 6 \mathrm{~T})$. The magnetic field was parallel to the optical excitation axis and the sample growth direction was rotated from the optical axis by an angle of $\theta$. The magnetic field was measured by a lowtemperature InAs Hall detector. The PL polarization was measured at $1.722 \mathrm{eV}$ in the reflection geometry with a photoelastic modulator (PEM) and a Glan-Thompson linear polarizer as a circular polarization analyzer and was detected by a GaAs photomultiplier together with a double monochromator and a two-channnel gated photon counter. For the time-resolved measurement, a quarter wave $(\lambda / 4)$ plate and a synchroscan streak camera were used instead of the PEM and the photomultiplier. The degree of circular polarization, $\rho$, is defined by $\rho=\left(I_{+}-I_{-}\right) /\left(I_{+}+I_{-}\right)$, where $I_{+}$and $I_{-}$are the intensities of the right $\left(\sigma^{+}\right)$and left $\left(\sigma^{-}\right)$circularly polarized PLs under linearly polarized pumping, respectively.

Magnetic-field-dependent circular polarization of PL in InP QDs strongly depends on the charging condition. When InP QDs are doped by one electron or two electrons under the electric bias of $U=-0.1 \mathrm{~V}$ and $U=+0.5 \mathrm{~V}$, respectively, the circular polarization of PL monotonously decreases in proportion to the longitudinal magnetic field (not shown). In both the cases, two electrons, one doped electron and one photocreated electron or two doped electrons, form spin singlet at the lowest quantum state and the thermal spin orientation between Zeeman levels of the hole solely determines the polarization of PL.

Under the electric bias of $U=-0.8 \mathrm{~V}$, quasiresonant excitation at $1.771 \mathrm{eV}$ produces the PL spectrum shown in Fig. 1(a) having small transverse acoustic (12 meV), longitudinal acoustic $(22 \mathrm{meV})$, and longitudinal optical (LO) phonon (43 meV) structures. The phonon structures arise as a result of competition between nonradiative tunneling of holes at the photoexcited excited state and the phonon-mediated relaxation from the quasiresonantly excited states in InP quantum dots. ${ }^{11-13}$ The PL polarization was measured at $1.722 \mathrm{eV}$ indicated in Fig. 1(a). The acoustic-phonon-mediated relaxation should participate in the energy relaxation by $49 \mathrm{meV}$ which is not equal to the LO phonon energy. The LOphonon-mediated relaxation is so fast that spin orientation is kept highly, while acoustic-phonon-mediated relaxation is so slow that spin orientation is lost considerably, consistent with the report on CdSe quantum dots. ${ }^{14}$ The linearly polarized excitation we used produces two sets of opposite spin polarizations and the spin polarization of each set is lost equally and considerably. As a result, two bright excitons are formed equally and two dark excitons are formed equally. The balance between two bright excitons is reflected by the observed zero circular polarization at $B=0$, shown in Fig. 1(c).

Under the electric bias of $U=-0.8 \mathrm{~V}$, InP QDs are neutral. The $\sigma^{+}$polarized PL intensity of neutral InP QDs en- 


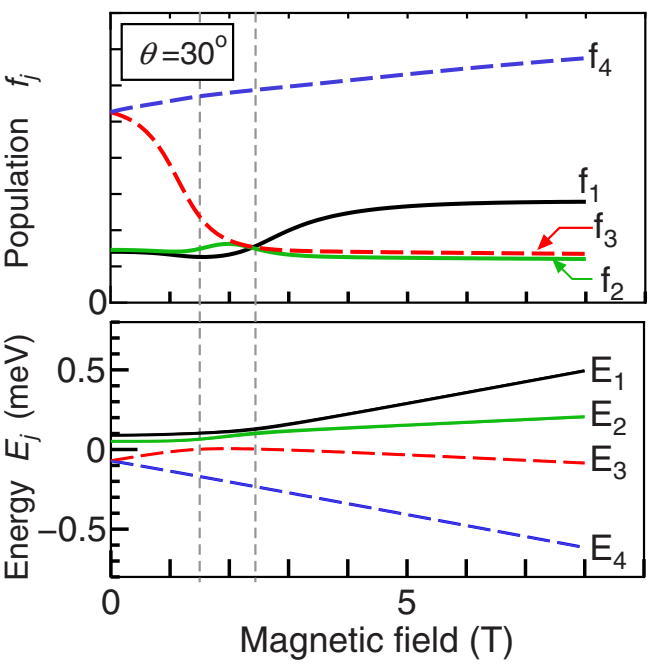

FIG. 2. (Color online) Calculated $f_{j}$ and $E_{j}$ at $\theta=30^{\circ}$ with parameters $g_{h, z}=1.02, \tau_{0} / \tau_{r}=10, \tau_{s} / \tau_{r}=10$, and $G_{r} / G_{0}=1$.

hances at 1.5 and $-2.5 \mathrm{~T}$ in the Faraday configuration at $5 \mathrm{~K}$, as is shown in Fig. 1. Simultaneously, the $\sigma^{-}$polarized PL intensity enhances at 2.5 and $-1.5 \mathrm{~T}$. Hence, $\rho$ has a dip (peak) at $2.5 \mathrm{~T}(-2.5 \mathrm{~T})$ and a peak (dip) at $1.5 \mathrm{~T}(-1.5 \mathrm{~T})$. The good agreement in crossing magnetic fields, 1.5 and $2.5 \mathrm{~T}$, between the calculation and the experiment shows that the resonant spin orientation takes place at the crossing points between bright and dark excitons. It also shows that $g_{h, z}=1.02$.

Spin polarization of excitons in semiconductors due to spin relaxation and sublevel mixing in the external magnetic field is discussed by Ivchenko and Kaminski1. ${ }^{15,16}$ Under the steady state, the population at the $|j\rangle$ state of the exciton, $f_{j}$, is determined by the following rate equation equalized to 0 :

$$
\frac{\partial f_{j}}{\partial t}=-\frac{1}{\tau_{j}} f_{j}-\sum_{j^{\prime} \neq j}\left(\frac{f_{j}}{T_{j^{\prime} j}}-\frac{f_{j^{\prime}}}{T_{j j^{\prime}}}\right)+G_{j}=0,
$$

where the first term in the second side describes the population decrease by the recombination, the second term the spin relaxation transfer between the $|j\rangle$ states of exciton, and the third term the generation rate of the $|j\rangle$ states of exciton. The recombination rate $1 / \tau_{j}$ is determined by nonradiative recombination rate $1 / \tau_{0}$ and radiative recombination rate $1 / \tau_{r}$ multiplied by the bright exciton component, $1 / \tau_{j}=\left(1 / \tau_{r}\right)\left(\left|C_{j, 1}\right|^{2}+\left|C_{j,-1}\right|^{2}\right)+1 / \tau_{0}$. The transfer rate $1 / T_{j^{\prime} j}$ between the $|j\rangle$ and $\left|j^{\prime}\right\rangle$ states of the exciton is given by $1 / T_{j^{\prime} j}=\left(w_{j^{\prime} j} / \tau_{s}\right) \Sigma_{m^{\prime} m} \eta_{m^{\prime} m}\left|C_{j, m} C_{j^{\prime} m^{\prime}}\right|^{2}$, where $\tau_{s}$ is the spin flip time, assuming spin flip of a single electron or hole alone $\left(\eta_{m^{\prime} m}=\delta_{\left|m-m^{\prime}\right|, l}, l=1,3\right) .{ }^{16}$ Here, the thermal Boltzmann distribution between the $|j\rangle$ and $\left|j^{\prime}\right\rangle$ states of the exciton is given by $w_{j^{\prime} j}=1$ for $E_{j^{\prime}}<E_{j}$ or $w_{j^{\prime} j}=\exp \left[-\left(E_{j^{\prime}}-E_{j}\right) / k_{B} T\right]$ for $E_{j^{\prime}}>E_{j}$. The generation rate for the $|j\rangle$ and $\left|j^{\prime}\right\rangle$ states of the exciton is given by $G_{j}=G_{r}\left(\left|C_{j, 1}\right|^{2}+\left|C_{j,-1}\right|^{2}\right)+G_{0}$, $G_{r} / G_{0}=1$ (Ref. 17) for linearly polarized quasiresonant excitation. After $f_{j}$ are obtained, $I_{ \pm}$are calculated by $I_{ \pm} \propto\left|C_{1, \pm 1}\right|^{2} f_{1}+\left|C_{2, \pm 1}\right|^{2} f_{2}+\left|C_{3, \pm 1}\right|^{2} f_{3}+\left|C_{4, \pm 1}\right|^{2} f_{4}$ and $\rho$ is calculated by $\rho=\left(I_{+}-I_{-}\right) /\left(I_{+}+I_{-}\right)$.

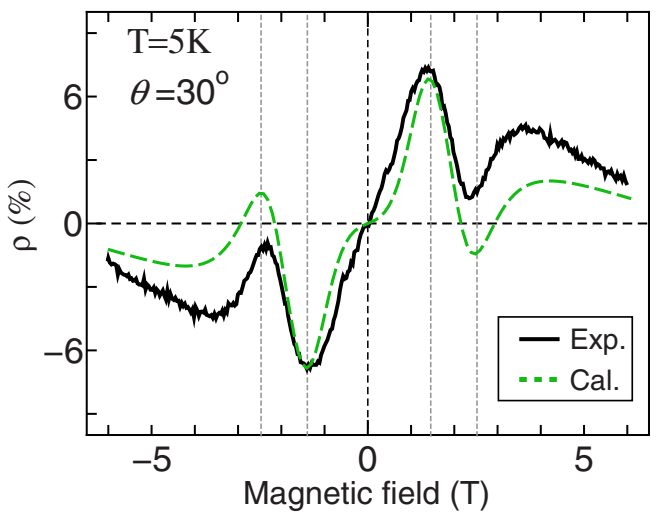

FIG. 3. (Color online) Circular polarization of luminescence of neutral InP quantum dots and calculated circular polarization under the tilted magnetic field at $\theta=30^{\circ}$. The parameters $g_{h, z}=1.02$, $\tau_{0} / \tau_{r}=10, \tau_{s} / \tau_{r}=10$, and $G_{r} / G_{0}=1$ (Ref. 17) were used.

The calculated $f_{j}$ are displayed together with $E_{j}$ as a function of the magnetic field for $\theta=30^{\circ}$ in Fig. 2. The existing probability for dark excitons is high because of their long lifetime. At the anticrossing points, the wave function of the dark exciton mixes with the wave function of bright excitons, and therefore, the more existing dark exciton luminesces through the bright exciton components, $\left|C_{j, \pm 1}\right|^{2}$. As a result, $\sigma^{+(-)}$polarized PL increases. The calculated $\rho$ is plotted in Fig. 3 and generally agrees with the experimental $\rho$. The resonant spin orientation structures for $\theta=30^{\circ}$ are observed at slightly smaller magnetic fields than 1.5 and $2.5 \mathrm{~T}$ because of anisotropy of the hole $g$ factor and increasing off-diagonal element in the Hamiltonian. The resonant spin orientation comes from increase of the luminescence of existing dark excitons. This means that the spin flip time is not shorter than the lifetime of the exciton and that dark excitons are existing more than bright excitons. Then, dark excitons work as the carrier and spin reservoir because of their long lifetime. With the increase of temperature, resonant spin orientation structure gradually decreased and disappeared at $65 \mathrm{~K}$. This temperature dependence is not explained by the thermalization between $|j\rangle, w_{j^{\prime} j}$, because $\left(E_{j^{\prime}}-E_{j}\right) / k_{B} T \ll 1$ holds for anticrossing pairs. Instead, it is explained by the decrease of the spin relaxation time $\tau_{s}$ at the elevated temperature. With the increase of temperature, $\tau_{s}$ decreases and dark excitons do not work as the spin reservoir because of frequent conversion between dark excitons and bright excitons. Calculation showed that decrease of $\tau_{s} / \tau_{r}$ to 1 resulted in $85 \%$ reduction in the resonant spin orientation structure. The decrease of the spin relaxation time $\tau_{s}$ at the elevated temperature was reported in our preceding papers. ${ }^{18,19}$

In longitudinal magnetic field $\left(\theta=0^{\circ}\right)$, bright excitons do not mix with dark excitons in the frame of the present spin Hamiltonian. Then, crossing does not give any change of the polarization on the theoretical model. However, precise $\theta$-dependent measurement with resolution of $0.1^{\circ}$ showed no decrease of the resonant spin orientation structure. This fact suggests that the spin Hamiltonian contains a small offdiagonal element except for the $\left(\mu_{B} B / 2\right) g_{e, x} \sin \theta$ and $\left(\mu_{B} B / 2\right) g_{h, x} \sin \theta$ terms, as is discussed previously. ${ }^{15}$ The 


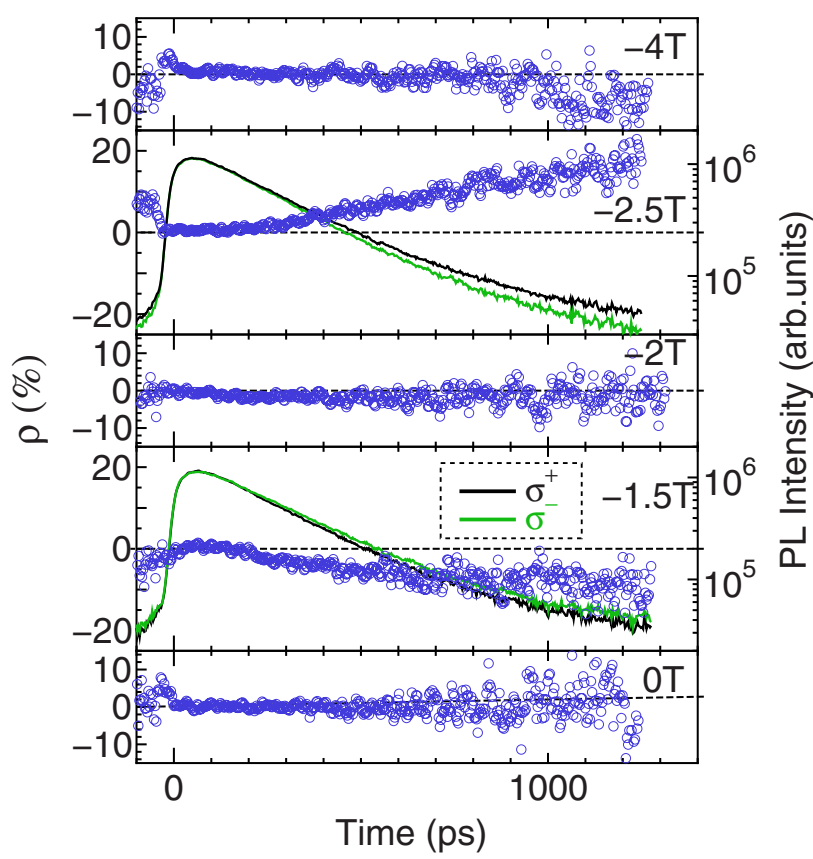

FIG. 4. (Color online) Temporal change of the $\sigma^{+}$and $\sigma^{-}$polarized luminescences (solid lines) and circular polarization $(\bigcirc)$ as a function of the longitudinal magnetic field at $5 \mathrm{~K}$.

off-diagonal element may come from lowered symmetry of InP dots and hyperfine interaction between electron and nuclear spins. If we assume a small off-diagonal element, $V_{13}=V_{24}=V_{31}=V_{42},{ }^{15}$ of $1.7 \mu \mathrm{eV}$, calculation shows that the resonant spin orientation structure for $\theta=0^{\circ}$ is narrowed but is not reduced in the amplitude. Fluctuated nuclear hyperfine field $B_{f}$ applied to electrons that we observed in InP QDs is $15 \mathrm{mT}$, which corresponds to energy change of $g_{e} \mu_{B} B_{f}$ $=1.7 \mu \mathrm{eV}$ for electrons. ${ }^{20}$ Therefore, hyperfine interaction between electron and nuclear spins as well as symmetry reduction of QDs from $C_{2 v}$ are the probable origin for the off-diagonal element causing the anticrossing for $\theta=0^{\circ}$. Then, bright excitons mix with dark excitons at $B=1.5$ and
$2.5 \mathrm{~T}$ and strong anticrossings and resonant spin orientation take place.

Temporal change of the circular polarization of PL is shown in Fig. 4 as a function of the longitudinal magnetic field. Circular polarization is almost constant at $B=0,-2$, and $-4 \mathrm{~T}$. It slowly decreases at $-1.5 \mathrm{~T}$, while it increases at $-2.5 \mathrm{~T}$ as time proceeds. The slow decrease and increase are explained by mixing of the bright and dark excitons at the anticrossing. At $-1.5 \mathrm{~T}(-2.5 \mathrm{~T}),|-1\rangle_{0}\left(|+1\rangle_{0}\right)$ state mixes with $|-2\rangle_{0}$ state, and $\sigma^{-(+)}$polarization increases as time proceeds because the contribution of more existing long-living dark exciton to the $\sigma^{-(+)}$polarized PL grows. At the -1.5 and $-2.5 \mathrm{~T}$, respectively, $\sigma^{-}$and $\sigma^{+}$polarizations are observed before the rise of PL. They are explained by the long lifetime of the dark exciton comparable to $12 \mathrm{~ns}$ of the laser repetition period. The decay time constant of PL is 260 ps, as is shown in Fig. 4. Suppose $\tau_{r}=260 \mathrm{ps}, \tau_{0} / \tau_{r}=10$ and $\tau_{s} / \tau_{r}=10$ give $\tau_{0}=2.6 \mathrm{~ns}$ and $\tau_{s}=2.6 \mathrm{~ns}$. The exciton spin relaxation time is much longer than the bright exciton lifetime, in consistent with previous study on QDs. ${ }^{21-23}$

In summary, resonant spin orientation of excitons was observed under linearly polarized quasiresonant excitation around two anticrossing points of excitons in InP QDs. Under the longitudinal and tilted magnetic fields of 1.5 and $2.5 \mathrm{~T}$, two anticrossings of bright and dark excitons take place. Then, bright and dark excitons mix with each other and the wave function mixing induces the resonant spin orientation. Time-resolved circular polarization clearly showed the resonant spin orientation due to mixing of bright and dark excitons at the anticrossing points. Dark excitons can work as the carrier and spin reservoir because of their long lifetime. We demonstrated that magnetic field can control the spin entanglement and the resonant spin orientation of QDs.

The authors thank I. A. Yugova and I. V. Ignatiev for helpful discussions on the anticrossing of bright and dark excitons and H.-W. Ren for the sample growth. This work was supported by Grant-in-Aid for Scientific Research No. 18204028 from the MEXT of Japan and "R\&D promotion scheme funding international joint research" promoted by NICT of Japan.

*shoichi@sakura.cc.tsukuba.ac.jp

${ }^{1}$ I. Žutić, J. Fabian, and S. Das Sarma, Rev. Mod. Phys. 76, 323 (2004).

${ }^{2}$ D. Loss and D. P. DiVincenzo, Phys. Rev. A 57, 120 (1998).

${ }^{3}$ A. V. Khaetskii and Y. V. Nazarov, Phys. Rev. B 61, 12639 (2000).

${ }^{4}$ P. G. Baranov, I. V. Mashkov, N. G. Romanov, P. Lavallart, and R. Planel, Solid State Commun. 87, 649 (1993); JETP Lett. 60, 446 (1994).

${ }^{5}$ E. Blackwood, M. J. Snelling, R. T. Harley, S. R. Andrews, and C. T. B. Foxon, Phys. Rev. B 50, 14246 (1994).

${ }^{6}$ M. Bayer, O. Stern, A. Kuther, and A. Forchel, Phys. Rev. B 61, 7273 (2000).

${ }^{7}$ I. A. Yugova, I. Ya. Gerlovin, V. G. Davydov, I. V. Ignatiev, I. E. Kozin, H. W. Ren, M. Sugisaki, S. Sugou, and Y. Masumoto,

Phys. Rev. B 66, 235312 (2002)

${ }^{8}$ Y. Masumoto, I. V. Ignatiev, K. Nishibayashi, T. Okuno, S. Yu. Verbin, and I. A. Yugova, J. Lumin. 108, 177 (2004).

${ }^{9}$ I. V. Ignatiev, T. Okuno, S. Yu. Verbin, I. A. Yugova, and Y. Masumoto, Physica E (Amsterdam) 17, 365 (2003).

${ }^{10}$ I. E. Kozin, V. G. Davydov, I. V. Ignatiev, A. V. Kavokin, K. V. Kavokin, G. Malpuech, H.-W. Ren, M. Sugisaki, S. Sugou, and Y. Masumoto, Phys. Rev. B 65, 241312(R) (2002).

${ }^{11}$ I. V. Ignatiev, I. E. Kozin, S. V. Nair, H.-W. Ren, S. Sugou, and Y. Masumoto, Phys. Rev. B 61, 15633 (2000).

${ }^{12}$ I. V. Ignatiev, I. E. Kozin, V. G. Davydov, S. V. Nair, J.-S. Lee, H.-W. Ren, S. Sugou, and Y. Masumoto, Phys. Rev. B 63, 075316 (2001).

${ }^{13}$ Semiconductor Quantum Dots: Physics, Spectroscopy and Applications, edited by Y. Masumoto and T. Takagahara (Springer, 
Berlin, 2002), Chap. 6.

${ }^{14}$ M. Scheibner, G. Bacher, S. Weber, A. Forchel, Th. Passow, and D. Hommel, Phys. Rev. B 67, 153302 (2003).

${ }^{15}$ E. L. Ivchenko and A. Yu. Kaminskiı̌, Phys. Solid State 37, 768 (1995).

${ }^{16}$ E. L. Ivchenko, Pure Appl. Chem. 67, 463 (1995).

${ }^{17}$ Under the quasiresonant excitation, bright excitons are more populated than dark excitons when excitons are formed because spin polarizations are partially preserved as a result of phononmediated relaxation. A setting of $G_{r} / G_{0}=1$, which means that the bright exciton population is twice of the dark exciton population initially, is consistent with the initial expected population ratio of bright and dark excitons. By varying the ratio $G_{r} / G_{0}$ from 1 to 0 , we found that the resonant spin orientation structure increased gradually by $70 \%$. In good consistency with the calculation, the resonant spin orientation structure observed under the above-barrier HeNe laser excitation was enhanced by $60 \%$.
The good consistency supports $G_{r} / G_{0}=1$ used for the present quasiresonant excitation.

${ }^{18}$ B. Pal, M. Ikezawa, Y. Masumoto, and I. V. Ignatiev, J. Phys. Soc. Jpn. 75, 54702 (2006).

${ }^{19}$ Y. Masumoto, S. Oguchi, B. Pal, and M. Ikezawa, Phys. Rev. B 74, 205332 (2006).

${ }^{20}$ B. Pal, S. Yu. Verbin, I. V. Ignatiev, M. Ikezawa, and Y. Masumoto, Phys. Rev. B 75, 125322 (2007).

${ }^{21}$ M. Paillard, X. Marie, P. Renucci, T. Amand, A. Jbeli, and J. M. Gérard, Phys. Rev. Lett. 86, 1634 (2001).

${ }^{22}$ Yu. G. Kusrayev, A. V. Koudinov, B. P. Zakharchenya, S. Lee, J. K. Furdyna, and M. Dobrowolska, Phys. Rev. B 72, 155301 (2005).

${ }^{23}$ S. Mackowski, T. A. Nguyen, T. Gurung, K. Hewaparakrama, H. E. Jackson, L. M. Smith, J. Wrobel, K. Fronc, J. Kossut, and G. Karczewski, Phys. Rev. B 70, 245312 (2004). 